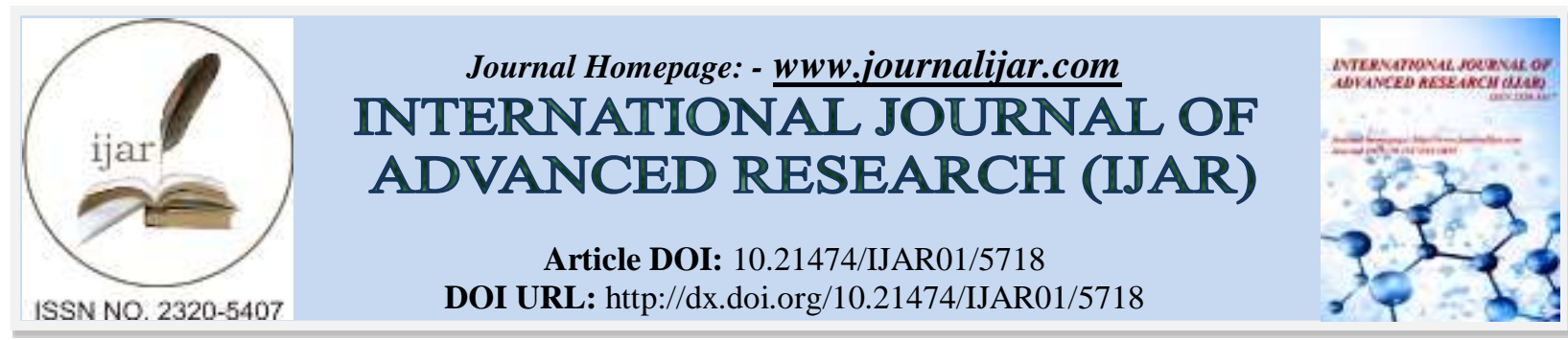

RESEARCH ARTICLE

\title{
MULTI-CRITERION ANALYSIS AND CARTOGRAPHY OF AREAS SENSITIVE TO SOIL EROSION IN TROPICAL URBAN ENVIRONMENTS: AN EXAMPLE OF ANYAMA (NORTHERN SUBURB OF ABIDJAN).
}

\begin{abstract}
ALLA Della André.
Maitre de Conférences, Institut de Géographie Tropicale (IGT), Université Félix Houphouët Boigny (Côte d'Ivoire).
\end{abstract}

\section{Manuscript Info}

Manuscript History

Received: 25 August 2017

Final Accepted: 27 September 2017

Published: October 2017

Key words:-

Anyama, Abidjan, vulnerability, risk of erosion, GIS.

\begin{abstract}
In the African cities south of the Sahara, soil erosion poses a significant challenge to policymakers and scientists alike. Most studies of this phenomenon focused their attention on of its impacts on spatial and socio-economic issues (Pain, 1984; Peyrot, 1984; Tchotsoua and Bonvallot, 1995 and 2000; Alla, 2010). An analysis identifying areas of soil erosion risks has not fully been investigated and yet it is paramount in order to make informed decision dealing with managing erosion.

Potentials for soil erosion result from a combination of several factors including nature and human made triggers. In a case study based on the city of Anyama, GIS was used to develop a model combining erosive natural factors (slope, composition of soil and types of soil protection), and anthropic elements such as build ups, building materials used, and density of population. To accomplish this, the Analytical Hierarchy Process (AHP), a multi-criteria method developed by Saaty (1984) was used. Each factor and variable were ranked from $1-5$ and the weigh of each factor was determined in the process. This enabled the analysis to map different zones of potential soil erosion. The results showed that in Anyama, 45\% of the urban space is identified as high risk for erosion, $36 \%$ as moderate risk and $19 \%$ as low-level risk for erosion. This result is in line with the use of scientific processes to support decision-making schemes by policymakers.
\end{abstract}

Copy Right, IJAR, 2017,. All rights reserved.

\section{Introduction:-}

La cartographie des zones à risque d'inondation, de glissement ou éboulement de terrain, ainsi que des zones d'érosion a connu une révolution spectaculaire avec le développement de l'informatique. Ainsi, dans les études relatives aux risques naturels où il faut souvent manipuler plusieurs données spatiales, les chercheurs ont de plus en plus recours au système d'information géographique (SIG). En effet, l'identification des phénomènes naturels créateurs de dommages, communément appelés aléas naturels, passe par la réalisation puis la superposition ou le croisement d'un certain nombre de cartes. Selon Atsé (2001), ces cartes traduisent ce que l'on sait ou croit savoir de la répartition et de la variation spatiale de certains facteurs de prédisposition à la manifestation de certains types de phénomènes.

Corresponding Author:- ALLA Della André.

Address:- Maitre de Conférences, Institut de Géographie Tropicale (IGT), Université Félix Houphouët 
C'est dans ce cadre que nous plaçons ce travail de recherche qui se veut une proposition de démarche méthodologique pour aider au mieux à déterminer les zones à risque d'érosion en milieu urbain, plus particulièrement en Afrique subsaharienne où la croissance des villes fait apparaitre que l'espace produit ne fait pas toujours l'objet d'un aménagement convenable. Ainsi, une bonne partie du sol est exposée à l'érosion. A cet effet, des études ont été menées à Kinshasa (Pain, 1984), à Brazzaville (Peyrot, 1984), à Yaoundé et dans d'autres villes du Cameroun (Tchotsoua M. et Bonvallot J., 1995, 2000), dans la périphérie d'Abidjan (Alla, 2010). Elles montrent que dans les pays tropicaux, l'urbanisation mal maîtrisée et parfois anarchique dans les quartiers périphériques favorise le ruissellement des eaux pendant les pluies. Ce ruissellement provoque l'érosion mécanique, facteur essentiel de dégradation des sites, de décapage du sol aux pieds des maisons et des infrastructures. Dans les rares cas où on aborde la répartition spatiale du risque d'érosion dans une recherche (par exemple, KOUADIO Boyossoro H., 2001 ; N'Goh, 2000), l'étude porte non seulement sur le milieu rural, mais aussi l'approche méthodologique n'est pas suffisamment poussée et les résultats ne facilitent pas la prise de décision, en termes de gestion du risque.

Pour contribuer à l'amélioration de la cartographie des zones à risque d'érosion et la rendre plus opérationnelle, cette étude prend appui sur le cas d'Anyama, ville de la périphérie nord d'Abidjan. En effet, Anyama, comme la plupart des villes ivoiriennes, se développe sans aménagement préalable sur tous les sites, ce qui est à l'origine d'une activité érosive dont les traces sont visibles dans l'espace. Comment cette érosion se répartit-elle ? Quels sont les paramètres qui influencent cette répartition et leur importance dans la manifestation de l'érosion à Anyama ?

L'approche choisie pour répondre à ces interrogations repose sur une analyse multicritère par laquelle les facteurs susceptibles d'intervenir dans la manifestation de l'aléa érosion et les éléments qui pourraient être endommagés subissent diverses combinaisons, en fonction de l'objectif visé.

\section{Description de la zone d'étude:- \\ I.1. Un site très accidenté:-}

Anyama est l'une des villes qui se sont développées dans la périphérie d'Abidjan. Peuplée de 148962 habitants $\left(\mathrm{RGPH}^{1}, 2014\right)$, elle est située entre $5^{\circ} 26^{\prime}-5^{\circ} 31^{\prime}$ de latitudes $\mathrm{N}$ et $4^{\circ} 2^{\prime}-4^{\circ} 5^{\prime}$ de longitudes $\mathrm{W}$ (figure 1) et se déploie sur un relief particulièrement accidenté. Il s'agit d'un plateau constitué par un interfluve allongé dans le sens nord-sud et coincé entre deux vallées profondes creusées par les affluents de la rivière Bété : celle de l'Apépo à l'ouest, encaissée d'environ $30 \mathrm{~m}$ et celle de l'Abouabo à l'est, d'une quarantaine de mètres de profondeur. Au sud, le rapprochement de ces deux vallées bloque l'allongement de l'interfluve. De même dans la partie nord, une grande échancrure en arc de cercle laissée par la Bété et ses ramifications délimite l'interfluve.

Au-delà de ce site principal, le relief présente deux paysages topographiques contrastés : à l'est et au sud, le plateau est très disséqué, réduisant les interfluves en des croupes convexes ; à l'ouest, il est plutôt faiblement découpé, avec des interfluves plus étendus, à surface légèrement ondulée et plus propices à l'urbanisation.

\section{I.2. Des sols peu résistants à l'érosion:-}

Anyama se développe sur des terrains taillés dans des sables argileux tertiaires, une formation sédimentaire plus ou moins épaisse qui constitue au nord de la lagune Ébrié le Continental terminal. Formés de sables à intercalations argileuses bariolées, les sables argileux sont des matériaux très homogènes tant au niveau latéral que vertical sur des profondeurs assez importantes (10 à 20 mètres ou plus). Dans ces formations où le pourcentage de la fraction argileuse est important, celle-ci croît avec la profondeur. Ce sont donc des terrains plus ou moins plastiques en fonction des proportions de sable et d'argile et qui, à l'état nu, n'opposent pas de résistance à l'impact des pluies.

\section{I.3. Des pluies importantes et agressives:-}

La zone d'Anyama est sous l'influence des pluies qui tombent dans les stations du Banco et d'Azaguié, avec des moyennes annuelles varient entre 1600 et $2000 \mathrm{~mm}$.

Ces pluies se concentrent sur deux saisons dont la plus longue va de mars à juillet. Au cours de cette saison, les pluies sont plus fréquentes et plus violentes (101 à $581 \mathrm{~mm}$ au Banco ; 146 à $304 \mathrm{~mm}$ à Azaguié). Elles atteignent leur maximum en juin, le mois le plus pluvieux de l'année. La seconde saison humide est plus courte et moins arrosée que la précédente. Elle couvre les mois de septembre à novembre, avec octobre qui reçoit chaque année entre 180 et $211 \mathrm{~mm}$ de pluie. C'est pendant ces deux saisons que le site urbain subit sa plus forte dégradation.

\footnotetext{
${ }^{1} \mathrm{RGPH}$ : Recensement général de la population et de l'habitat
} 


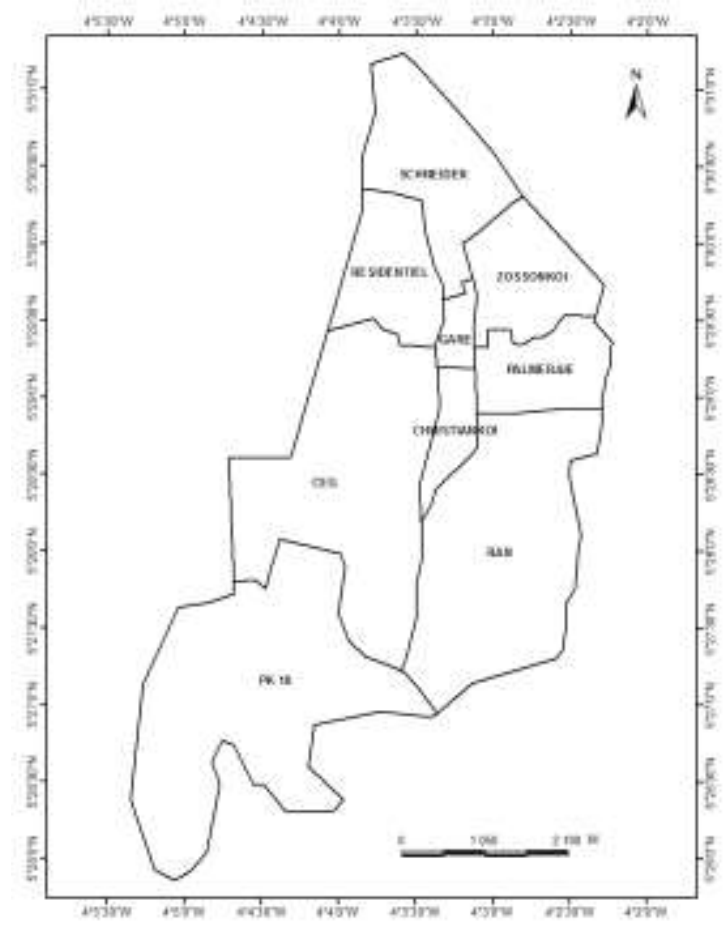

Figure 1 :- Présentation de la ville d'Anyama

\section{Données et méthodes:-}

Quelles sont les données dont on a besoin pour mener une telle étude ? Comment les traiter pour avoir des résultats fiables?

\section{II.1. Données utilisées et définition des couches d'information:-}

Le choix des données est capital dans l'élaboration des cartes des zones à risques. Il est fonction du type de risque que l'on veut identifier. Dans le cas des zones où l'érosion serait susceptible de se produire, il s'agit des documents qui vont servir à extraire des couches d'information dont la superposition ou le croisement permet de traduire la variation spatiale des facteurs de prédisposition à la manifestation de l'érosion (aléa) et de ceux relatifs aux éléments exposés (enjeux) :

- Les photographies aériennes ou les images satellites mettent en évidence les formes d'occupation de l'espace à un moment donné. Pour Anyama, nous avons utilisé les images Quickbird de 2013 qui ont permis de délimiter l'espace urbanisé, de déterminer la densité des constructions (le bâti), la qualité des constructions (le bâti) sur la base des types d'habitat et le niveau de protection du sol urbain contre l'érosion. La définition de cette dernière couche s'est appuyé sur le niveau d'aménagement de l'espace urbain (bitumage, réseau de drainage, engazonnement, etc.) ;

- La carte géotechnique à 1/50 000 d'Abidjan a permis d'extraire la couche des limites des faciès géologiques de la zone d'Anyama;

- La carte topographique d'équidistance de $2 \mathrm{~m}$, présentant une bonne lisibilité des courbes de niveau, permet une analyse plus fine des formes de relief. De cette carte, a été extraite la couche «pente »;

- Les données démographiques sont importantes pour évaluer l'endommagement potentiel des personnes (enjeux humains). Celles issues du recensement de 2014, disponibles à l'échelle des quartiers, ont servi à créer la couche «densité de population».

- Pour les données pluviométriques, facteur déterminant dans le déclenchement de l'aléa érosion, les informations importantes sont les pluies journalières. Dans une ville comme Anyama où les pluies sont partout élevées et donc susceptibles de provoquer l'érosion dans tous les secteurs urbains, l'agressivité climatique est considérée comme invariable. 


\section{II.2. Etapes de la modélisation et de la cartographie des zones à risque d'érosion:-}

Elles sont relatives à la façon dont les couches d'informations sont manipulées et combinées pour élaborer les cartes des zones d'aléa érosion, des niveaux d'enjeux et des zones à risque d'érosion (figure 2).

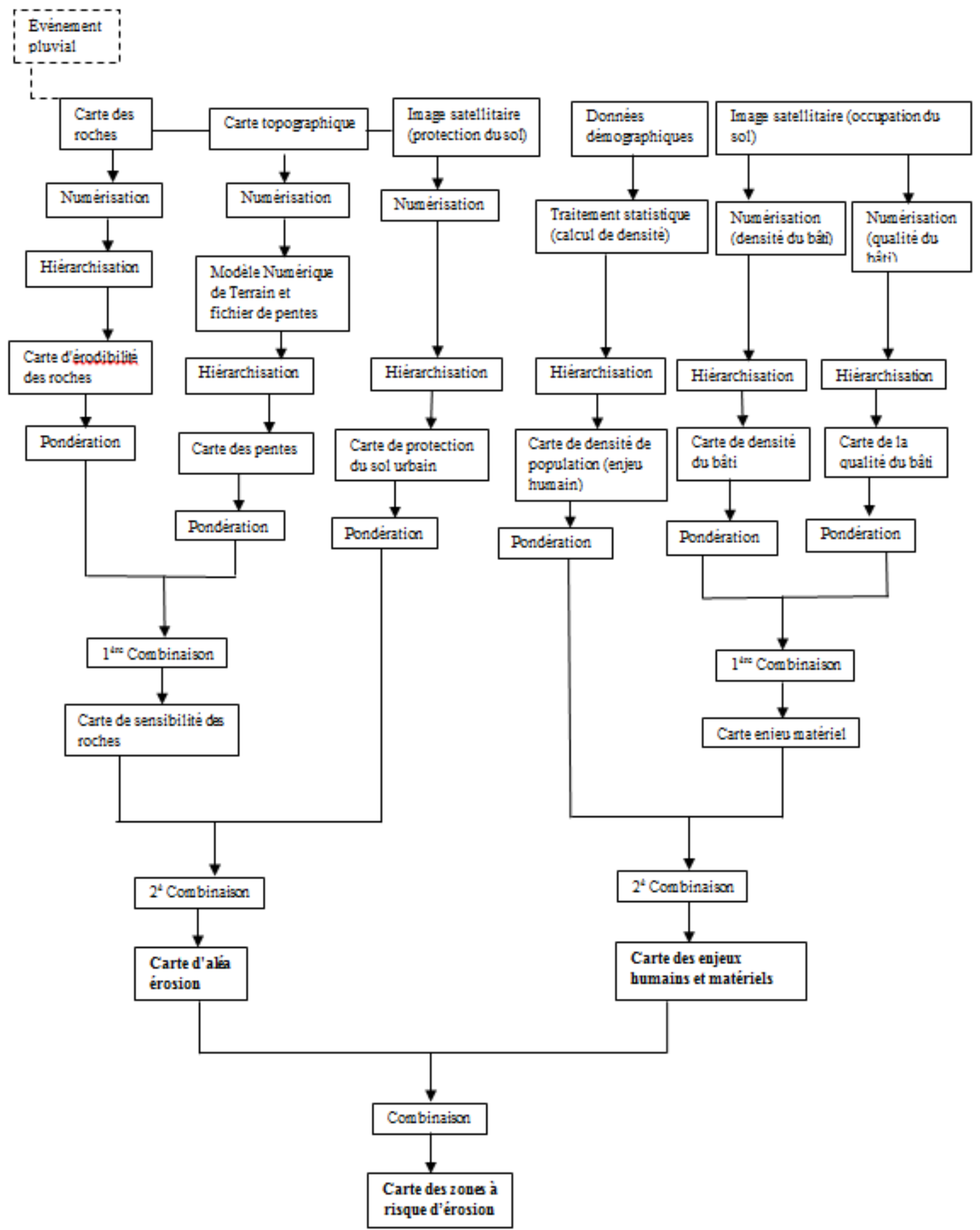

Figure 2:- Les étapes de la cartographie des zones à risque d'érosion en milieu urbain 


\section{II.2.1. Détermination des zones d'aléa érosion}

Pour identifier les zones prédisposées à l'aléa érosion à Anyama, on s'est appuyé sur les facteurs dont la combinaison permet de déterminer les zones où l'érosion peut se manifester ou se manifeste effectivement dans la ville. Il s'agit de la pente, de la protection du sol et de l'érodibilité des sols, l'agressivité des pluies étant considérée comme uniforme à l'échelle de la ville. Les cartes liées à ces facteurs ont été élaborées en fonction des niveaux d'exposition à l'aléa érosion. La première étape de cette opération est la hiérarchisation des facteurs.

Hiérarchisation des facteurs de l'aléa

L'appréciation de l'aléa érosion commence par la hiérarchisation des facteurs qui y contribuent, c'est-à-dire la pente, la protection du sol et l'érodibilité des sols. Pour chaque facteur, la gamme des variations de valeurs est divisée en classes. Chaque classe est affectée d'une note qui permet de situer les zones retenues dans l'étude sur des échelles de valeurs correspondant aux niveaux d'exposition à l'érosion. Le système de notation ainsi adopté est celui de la progression ordinale, de sorte que les points d'un facteur puissent être additionnés avec ceux des classifications relatives aux autres facteurs. Ces notes varient de 1 (indice nul) à 5 (indice très élevé) pour tous les facteurs. Chacune de ces échelles se présente de la façon suivante (tableau 1).

Tableau 1:- Notes attribuées aux indices de facteurs d'aléa érosion

\begin{tabular}{|l|l|}
\hline Indice des facteurs & Note \\
\hline Très faible & 1 \\
Faible & 2 \\
Moyen & 3 \\
Élevé & 4 \\
Très élevé & 5 \\
\hline
\end{tabular}

A Anyama, en tenant compte de la grande dissection du site et des contraintes d'aménagement des terrains urbains qui varient en fonction de l'importance des pentes (Akré, 2002), on a déterminé les classes de pentes sur la base de leur susceptibilité à l'érosion:

- $\quad$ zone de faible érosion (pentes inférieures à $5 \%$ ) ;

- zone d'érosion moyenne (pentes de 5 à $7 \%$ ) ;

- $\quad$ zone de forte érosion (pentes de 7 à $15 \%$ ) ;

- $\quad$ zone de très forte érosion (pentes de plus de $15 \%$ ).

Des valeurs relatives au potentiel érosif (notes) sont attribuées aux différentes classes de pente. Elles vont de 2 pour les pentes inférieures à $5 \%$, à 5, là où elles sont supérieures à $15 \%$.

$\mathrm{Au}$ niveau du sol, une seule roche sédimentaire, formée de sables argileux du Tertiaire, de résistance moyenne à l'érosion, constitue le substrat de l'espace actuellement urbanisé. Sa note est de 3.

Concernant les niveaux de protection du sol contre l'érosion définis à partir de l'interprétation des images satellites, ils varient à Anyama de nul sur les terrains dont la dénudation accroit la sensibilité à l'érosion (note 5) à élevé, là où le sol bénéficie d'une bonne protection (note 2).

Pondération des facteurs d'aléa érosion

L'objectif de la pondération est d'attribuer à chacun des facteurs (pente, roche et protection du sol) un "poids" qui corresponde à l'importance du rôle qu'il joue dans la manifestation de l'aléa érosion. Pour y arriver, nous nous sommes inspiré de la Méthode de Hiérarchie Multicritère (MHM) de Saaty (1984) dont l'application se fait en trois étapes.

La première étape : la construction de la matrice et l'établissement des priorités. Dans le cas du processus érosif, la matrice permet de comparer les facteurs d'aléa entre eux. Cette comparaison est binaire et permet d'obtenir un classement des facteurs selon leur importance relative dans le développement de l'érosion. Pour remplir la matrice, on attribue des valeurs numériques à des jugements subjectifs sur l'importance d'un facteur par rapport à un autre. Ces jugements s'appuient sur les données du tableau suivant. 
Tableau 2:- Correspondance des appréciations

\begin{tabular}{|l|l|l|}
\hline $\begin{array}{l}\text { Degré } \\
\text { d'importance }\end{array}$ & Céfinition & Commentaire \\
\hline 1 & Importance égale de deux éléments & Deux éléments contribuent autant à la propriété \\
\hline 3 & $\begin{array}{l}\text { Faible importance d'un élément par } \\
\text { rapport à l'autre }\end{array}$ & $\begin{array}{l}\text { L'expérience et l'appréciation personnelle favorisent } \\
\text { légèrement un élément par rapport à un autre }\end{array}$ \\
\hline 5 & $\begin{array}{l}\text { Importance forte ou déterminante d'un } \\
\text { élément par rapport à un autre }\end{array}$ & $\begin{array}{l}\text { L'expérience et l'appréciation personnelle favorisent } \\
\text { fortement un élément par rapport à un autre }\end{array}$ \\
\hline 7 & $\begin{array}{l}\text { Importance attestée d'un élément par } \\
\text { rapport à un autre }\end{array}$ & $\begin{array}{l}\text { Un élément est fortement favorisé et sa dominance est } \\
\text { attestée dans la pratique }\end{array}$ \\
\hline 9 & $\begin{array}{l}\text { Importance absolue d'un élément par } \\
\text { rapport à un autre }\end{array}$ & $\begin{array}{l}\text { Les preuves favorisant un élément par rapport à un } \\
\text { autre sont aussi convaincantes que possible }\end{array}$ \\
\hline $2,4,6,8$ & $\begin{array}{l}\text { Valeurs intermédiaires entre deux } \\
\text { appréciations voisines }\end{array}$ & Un compromis est nécessaire entre deux appréciations \\
\hline
\end{tabular}

Source : Saaty, 1984.

Les appréciations tiennent compte des travaux de recherche et de la culture scientifique du chercheur. En référence à ses connaissances, il émet des jugements relativement au processus érosif. Dans le cas d'Anyama, par rapport à la pente, la protection du sol a une importance déterminante. Elle prend la valeur 5. Comparée à la roche, la protection du sol a plutôt une importance attestée, ce qui lui vaut la valeur 7. Entre la roche qui est la même sur tout l'espace urbain (sables argileux) et la pente, c'est la pente qui a plus d'importance dans l'érosion. On lui attribue la valeur 4. L'attribution de valeurs aux appréciations doit se faire avec la plus grande objectivité possible, en gardant à l'esprit l'objectif poursuivi qui est ici la contribution de chacun des facteurs à la détermination de l'aléa érosion.

Une fois les valeurs choisies, on remplit la matrice (tableau 3). Pour cela, chaque facteur de la colonne de gauche est comparé successivement à chaque facteur de la ligne du haut de la matrice en fonction de l'objectif qui est le processus d'érosion. Si la comparaison est favorable au facteur de la colonne de gauche par rapport au facteur de la ligne, l'appréciation est exprimée à l'aide d'un entier (4 ou 5 par exemple); dans le cas contraire, elle est exprimée à l'aide d'une fraction (1/4 ou 1/5 par exemple). Le résultat d'un facteur comparé à lui-même prend la valeur 1.

Tableau 3:- Matrice de comparaison par paire des facteurs d'érosion à Anyama

\begin{tabular}{|l|l|l|l|}
\hline Processus érosif & Roche & Pente & Protection du sol \\
\hline Roche & 1 & $1 / 4$ & $1 / 7$ \\
\hline Pente & 4 & 1 & $1 / 5$ \\
\hline Protection du sol & 7 & 5 & 1 \\
\hline
\end{tabular}

En nous appuyant sur cette matrice, on détermine les vecteurs de priorité de chaque facteur. Pour ce faire, on procède à une série d'opérations:

- la sommation de chaque colonne ;

- la normalisation de la matrice qui consiste à diviser chaque valeur d'appréciation d'une colonne par la somme de cette colonne ;

- le calcul de la moyenne de chaque ligne, ce qui permet d'obtenir la priorité de chaque facteur, en fonction du processus d'érosion. La somme des priorités doit être égale à 1 ou $100 \%$.

A la fin de ces opérations, on a déterminé les vecteurs de priorité de chaque facteur d'aléa érosion : 0,08 pour le facteur "roche", 0,21 pour le facteur "pente" et 0,71 pour le facteur "protection du sol". Cela voudrait dire que dans le processus érosif à Anyama, la roche joue pour $8 \%$, la pente pour $21 \%$ et la protection du sol intervient de façon incontestable avec $71 \%$.

La deuxième étape est la cohérence logique. C'est la démarche par laquelle on teste la cohérence des appréciations. En effet, un raisonnement est dit cohérent si son indice de cohérence est inférieur ou égal à $10 \%$.

Pour déterminer l'indice de cohérence de la matrice d'appréciation des facteurs d'érosion, on multiplie d'abord les appréciations de chaque colonne par la priorité lui correspondant, puis on fait la somme des valeurs de chaque ligne. Ensuite, on divise chaque total de ligne par la priorité du facteur correspondant à cette ligne et on fait la moyenne 
des résultats précédents. Enfin, on détermine l'indice de cohérence : IC= $\frac{\text { Moyenne-nombre colonnes }}{\text { nombre colonnes }-1}$. Plus l'indice de cohérence descend en dessous de $10 \%$, plus le raisonnement utilisé pour la matrice de comparaison des facteurs d'érosion est cohérent. C'est le cas à Anyama où il est $\frac{3,11-3}{2}=0,055$ ou 5,5\%.

La troisième étape est la pondération des facteurs qui consiste à affecter à chaque facteur d'érosion son coefficient de pondération pour tenir compte de l'importance qu'on lui accorde dans le développement de l'aléa érosion. Dans ce cas, les notes attribuées aux classes de pente, de sol et de protection du sol urbain définies plus haut sont multipliées par la priorité de chaque facteur.

Le résultat est un modèle mathématique qui se présente sous la forme d'une équation de type : $\mathrm{AE}=0,08 \mathrm{nr}+0,21 \mathrm{np}$ $+0,71 \mathrm{nps}$, avec $\mathrm{AE}=$ aléa érosion $; \mathrm{n}=$ notes affectées aux classes des facteurs d'aléa érosion $; \mathrm{r}=$ roche $; \mathrm{p}=$ pente du relief ; ps = protection du sol.

Etablissement de la carte d'aléa érosion

Dans cette étape, une première combinaison est faite entre les notes pondérées des facteurs pente et types de sol. Les zones résultantes sont appelées zones sensibles à l'érosion ; elles ont comme valeur théorique du potentiel érosif la somme des valeurs de la classe de pente et du type de sol correspondants (tableau 4).

Tableau 4:- Indice de sensibilité des roches d'Anyama à l'érosion

\begin{tabular}{|l|l|l|l|l|l|l|}
\hline & \multicolumn{5}{|l|}{ Pente } \\
\hline & & $\leq 2 \%$ & 2 à $5 \%$ & 5 à $7 \%$ & 7 à15\% & $>15 \%$ \\
\hline Type de roche & Note pondérée & $\mathbf{0 , 2 1}$ & $\mathbf{0 , 4 2}$ & $\mathbf{0 , 6 3}$ & $\mathbf{0 , 8 4}$ & $\mathbf{1 , 0 5}$ \\
\hline Sables argileux du Tertiaire & $\mathbf{0 , 2 4}$ & 0,45 & 0,66 & 0,87 & 1,08 & 1,29 \\
\hline
\end{tabular}

En interprétant les valeurs ainsi obtenues, nous avons procédé à la définition de trois classes de zones sensibles à l'érosion dans la ville d'Anyama :

- $\quad$ indice $<0,70:$ sensibilité faible ;

- $\quad$ indice de 0,70 à $1:$ sensibilité moyenne ;

- $\quad$ indice $\geq 1:$ sensibilité élevée

Ensuite, une seconde combinaison permet de passer à la carte de l'aléa érosion. Celle-ci résulte de l'addition des indices de sensibilité des zones à l'érosion avec les notes pondérées du facteur protection du sol (tableau 5). Les valeurs issues de ce croisement donnent lieu à une couche d'information qu'il faut également examiner attentivement pour déterminer les classes correspondant aux niveaux d'aléa érosion dans différentes zones d'Anyama.

Tableau 5:- Indice de l'aléa érosion à Anyama

\begin{tabular}{|c|c|c|c|c|c|}
\hline \multirow{3}{*}{ 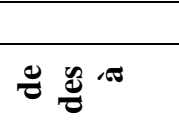 } & \multirow[b]{3}{*}{ Note pondérée } & \multicolumn{4}{|c|}{ Niveau de protection du sol } \\
\hline & & Élevé & Moyen & Faible & Nul \\
\hline & & 1,42 & 2,13 & 2,84 & $\mathbf{3 , 5 5}$ \\
\hline \multirow{5}{*}{ 莺 } & $\mathbf{0 , 4 5}$ & 1,87 & 2,58 & 3,29 & 4 \\
\hline & 0,66 & 2,08 & 2,79 & 3,5 & 4,21 \\
\hline & $\mathbf{0 , 8 7}$ & 2,29 & 3 & 3,71 & 4,42 \\
\hline & 1,08 & 2,5 & 3,21 & 3,92 & 4,63 \\
\hline & 1,29 & 2,71 & 3,42 & 4,13 & 4,84 \\
\hline
\end{tabular}

L'analyse de l'information contenue dans ce tableau a permis de dégager trois zones d'aléa érosion :

- $\quad$ indice $<2,80:$ zone d'aléa érosion faible ;

- $\quad$ indice de 2,80 à $3,80:$ zone d'aléa érosion moyen ;

- $\quad$ indice $\geq 3,80$ : zone d'aléa érosion élevé.

\section{II.2.2. Définition des niveaux d'enjeux présents dans la ville d'Anyama:-}

En milieu urbain, les enjeux sont nombreux et peuvent être plus ou moins importants, en fonction de leur répartition. En effet, les foyers de vulnérabilité qui mettent en évidence l'inégalité des espaces face aux risques sont dus à la concentration spatiale des enjeux. 
Dans le cas de cette étude sur l'érosion, pour déterminer les niveaux d'enjeux à Anyama, ont été retenus les enjeux humains, avec comme critère la densité de population, et les enjeux matériels où l'accent est mis sur la qualité des constructions (type de construction en fonction des matériaux utilisés) et sur leur densité (niveau de regroupement ou de dispersion).

Une fois que les critères sont connus, nous avons suivi le même principe que dans le cas des facteurs d'aléa érosion. Il est d'abord procédé à la hiérarchisation des enjeux dans laquelle les valeurs de chaque critère sont divisées en classes dont chacune est affectée d'une note allant de 1 à 5 qui exprime l'importance des enjeux ou leur niveau de vulnérabilité. Ensuite on est passé à la pondération des critères, en suivant les étapes de la hiérarchie multicritère ; ce qui a permis dans le cas d'Anyama d'aboutir à la formule suivante : Ehm $=0,08 \mathrm{ndp}+0,19 \mathrm{ndb}+0,73 \mathrm{nqb}$, dans laquelle Ehm = enjeux humains et matériels $; \mathrm{n}=$ la note affectée aux classes des critères d'enjeux ; dp = la densité de population ; $\mathrm{db}=$ la densité du bâti $; \mathrm{qb}=$ la qualité du bâti.

A partir de cette formule, la carte des enjeux matériels est réalisée. Celle-ci résulte du croisement des critères "densité" et "qualité" du bâti, avec comme valeurs la somme des notes pondérées des indices d'enjeux de chacun des deux critères (tableau 6).

Tableau 6:- Indice des enjeux matériels

\begin{tabular}{|l|l|l|l|l|}
\hline \multirow{2}{*}{} & \multirow{2}{*}{} & \multicolumn{2}{l}{ Indice d'enjeu (qualité du bâti) } \\
\cline { 3 - 5 } & & Faible & Moyen & Élevé \\
\hline Indice d'enjeu (densité du bâti) & Note pondérée & $\mathbf{1 , 4 6}$ & $\mathbf{2 , 1 9}$ & $\mathbf{2 , 9 2}$ \\
\hline Très faible & $\mathbf{0 , 1 9}$ & 1,65 & 2,38 & 3,11 \\
\hline Faible & $\mathbf{0 , 3 8}$ & 1,84 & 2,57 & 3,30 \\
\hline Moyen & $\mathbf{0 , 5 7}$ & 2,03 & 2,76 & 3,49 \\
\hline Élevé & $\mathbf{0 , 7 6}$ & 2,22 & 2,95 & 3,68 \\
\hline Très élevé & $\mathbf{0 , 9 5}$ & 2,41 & 3,14 & 3,87 \\
\hline
\end{tabular}

L'analyse de ces valeurs a permis de déterminer trois classes d'enjeux matériels :

- $\quad$ indice $<2,50$ : enjeux matériels faibles ;

- $\quad$ indice de 2,50 à 3,12: enjeux matériels moyens ;

- $\quad$ indice $\geq 3,12$ : enjeux matériels importants.

Les résultats de ce premier croisement ont été additionnés avec les notes pondérées du critère "densité de population" (tableau 7).

Tableau 7:- Indice des enjeux humains et matériels

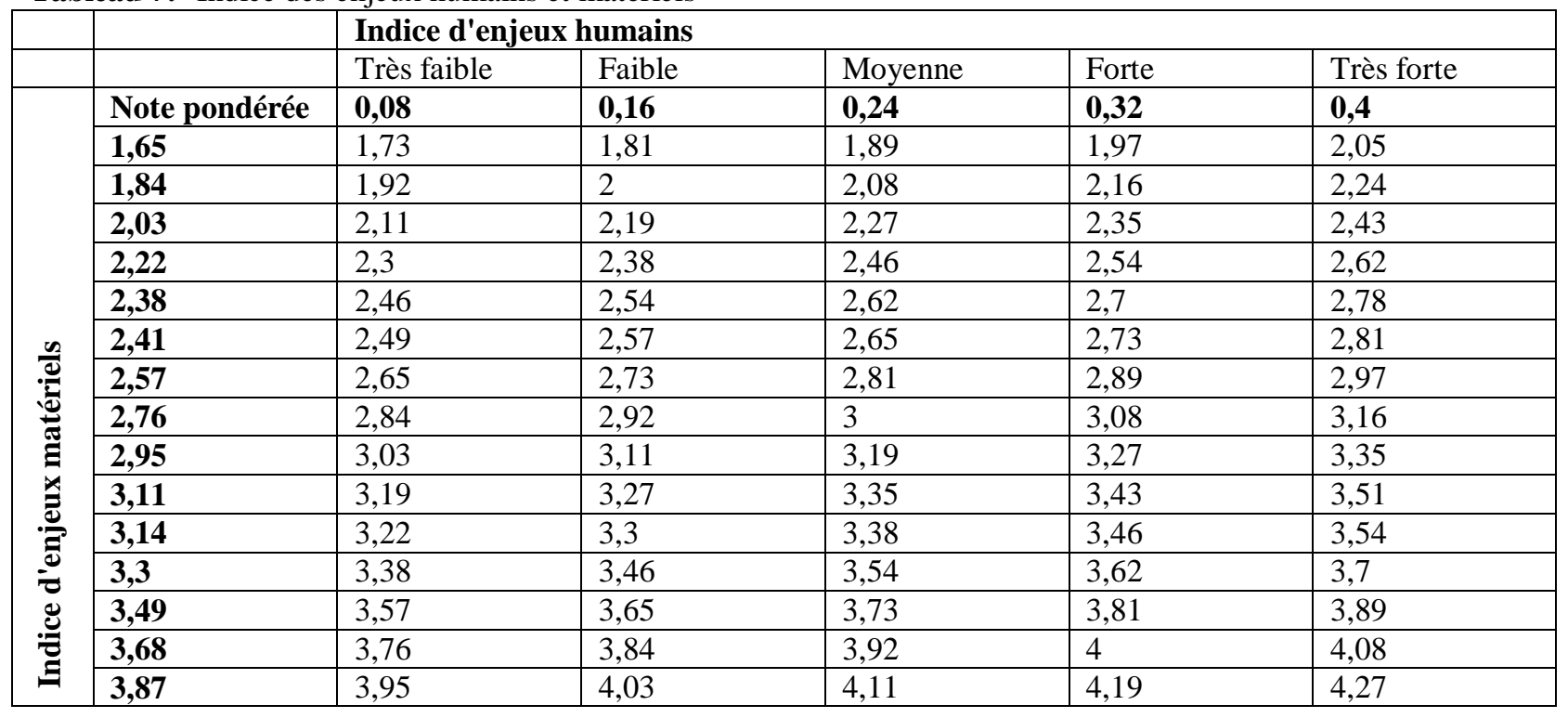


Un examen minutieux des données de ce tableau a permis de définir trois classes d'enjeux humains et matériels :

- $\quad$ indice $<2,80$ : enjeux humains et matériels faibles ;

- $\quad$ indice de 2,80 à 3,40 : enjeux humains et matériels moyens ;

- $\quad$ indice $\geq 3,40$ : enjeux humains et matériels importants.

\section{II.2.3. La détermination des zones à risque d'érosion:-}

La phase finale de la démarche est d'inventorier à l'intérieur de la ville d'Anyama les zones où l'érosion serait en mesure de créer des dommages en cas de manifestation. Elle repose sur la combinaison des couches d'information "aléa érosion" et "enjeux humains et matériels" et s'exprime par l'équation : RE (risque d'érosion) $=(0,08 \mathrm{nr}+$ $0,21 \mathrm{np}+0,71 \mathrm{nps}) \mathrm{x}(0,08 \mathrm{ndp}+0,19 \mathrm{ndb}+0,73 \mathrm{nqb})$. À travers le niveau d'aménagement du sol urbain et la qualité du bâti, la vulnérabilité, variable importante dans l'appréciation des niveaux de risque, est implicitement prise en compte.

Sous ArcMap, les résultats de cette combinaison sont stockés dans la table attributaire du fichier d'Anyama où la multiplication des valeurs pondérées de l'aléa érosion par celles des enjeux donne lieu à une base de données statistique. Cette base de données a été imprimée sous Excel et, en s'appuyant sur la matrice de combinaison qui suit (tableau 8), elle a servi à définir les niveaux de risque correspondants, ce qui a permis d'élaborer la carte des zones à risque d'érosion dans la ville d'Anyama.

Tableau 8:- Définition du risque en fonction de l'aléa et des enjeux (Inspiré de Yannick Manche, 2000)

\begin{tabular}{|l|l|l|l|l|l|}
\hline & $\begin{array}{l}\text { Enjeux très } \\
\text { importants }\end{array}$ & $\begin{array}{l}\text { Enjeux } \\
\text { importants }\end{array}$ & $\begin{array}{l}\text { Enjeux } \\
\text { moyens }\end{array}$ & Enjeux faibles & $\begin{array}{l}\text { Enjeux très } \\
\text { faibles }\end{array}$ \\
\hline Aléa très élevé & Très élevé & Très élevé & Élevé & Moyen & Faible \\
\hline Aléa élevé & Très élevé & Élevé & Élevé & Moyen & Faible \\
\hline Aléa moyen & Élevé & Élevé & Moyen & Moyen & Faible \\
\hline Aléa faible & Moyen & Moyen & Moyen & Faible & Très faible \\
\hline Aléa très faible & Faible & Faible & Faible & Très faible & Très faible \\
\hline
\end{tabular}

Ainsi, l'évaluation du risque d'érosion résulte du croisement de plusieurs données, par une corrélation spatiale sur un support cartographique de type SIG. Autrement dit, en effectuant un traitement spatial de l'information, on détermine les zones qui se situent dans un secteur soumis simultanément à l'aléa et aux enjeux.

\section{Résultats : les cartes et leur analyse:- \\ III.1. La carte d'aléa érosion:-}

Les zones sensibles à l'érosion à Anyama résultent de la combinaison spatiale par addition des valeurs pondérées des pentes et de la roche. Dans cette ville où le site est très accidenté, les terrains de sensibilité élevée dominent, ce qui indique qu'on est dans un espace qui réunit des attributs favorables à l'érosion : un sol moyennement ou peu résistant sur des pentes fortes. Une analyse statistique fait apparaître à ce sujet que sur une superficie urbaine de 739 ha, les zones de sensibilité élevée s'étendent sur 368 ha ; celles de faible sensibilité couvrent 254 ha et celles de sensibilité moyenne 126 ha (figure 3 ).

Le croisement des niveaux de sensibilité avec la protection du sol permet d'obtenir la carte de l'aléa érosion (figure 4). Celle-ci met en évidence la susceptibilité réelle des secteurs urbains à l'érosion du sol. Dans cette application de type SIG où chaque facteur est affecté de son poids dans le processus érosif, une analyse minutieuse de la carte permet de confirmer le rôle prééminent de la protection du sol dans le processus érosif. En effet, dans cette ville où l'urbanisation est de type populaire ou précaire, avec une quasi absence de contrôle de l'occupation du sol, les zones d'aléa élevé (304 ha) correspondent aux quartiers où la forte densification de l'habitat a fini par mettre les sols à nu. Les zones où l'aléa érosion est moyen ou faible concernent par contre les secteurs en cours d'urbanisation, en particulier les quartiers RAN et Résidentiel. 


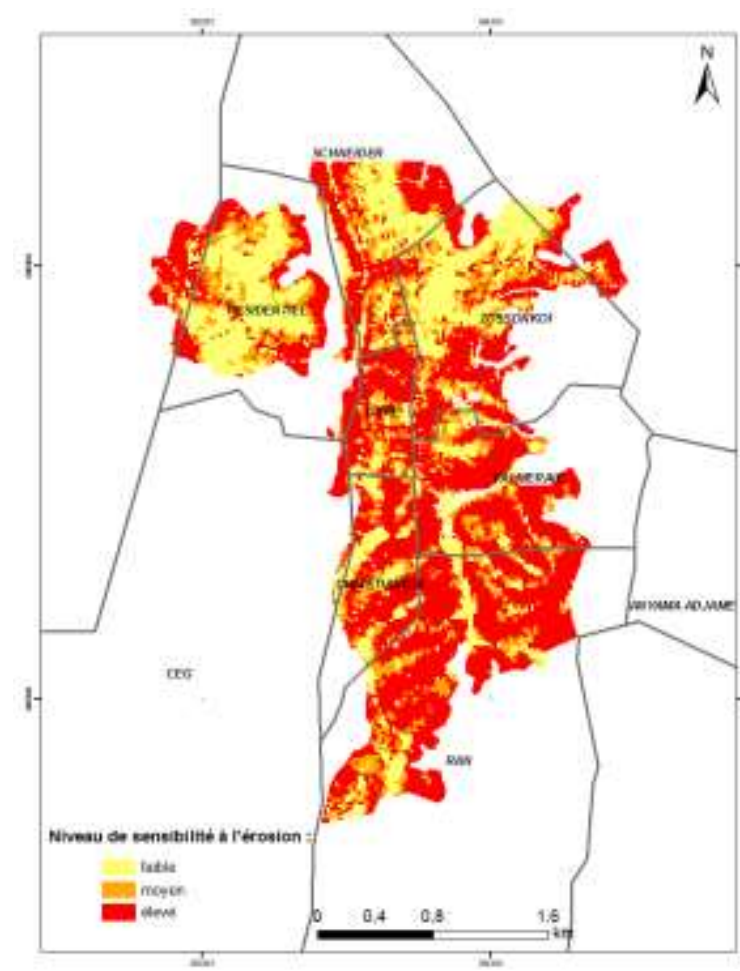

Figure3:- Répartition des terrains d'Anyama en fonction de leur sensibilité à l'érosion

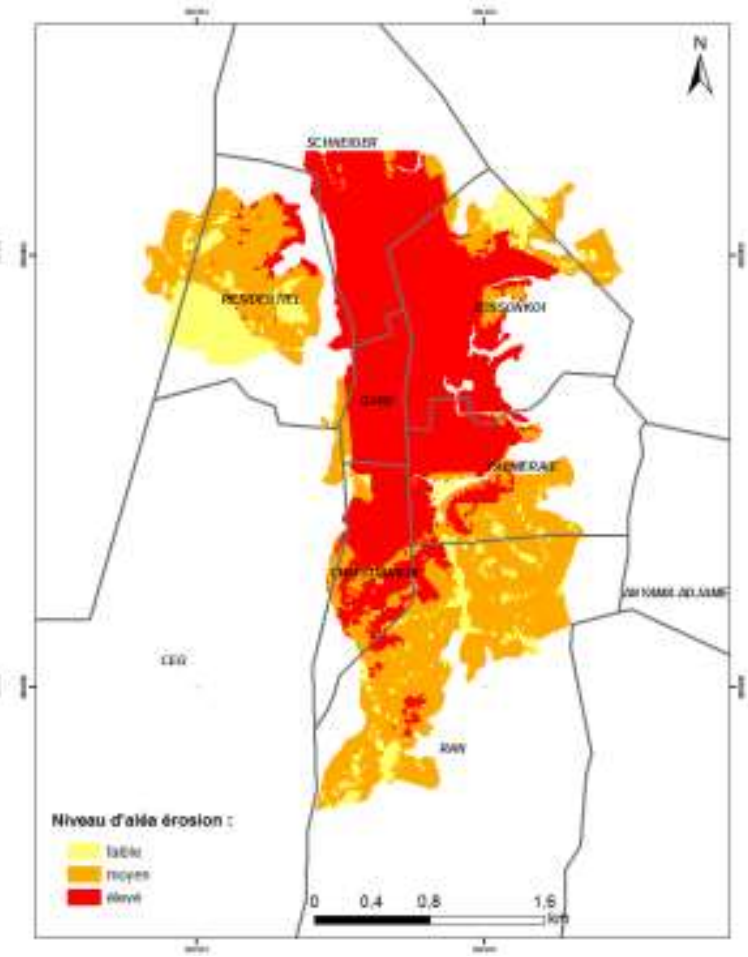

Figure4:- Répartition de l'aléa érosion dans la ville d'Anyama

\section{III.2. La carte de répartition des enjeux:-}

Elle résulte, dans un premier temps, du croisement des couches "densité du bâti" et "qualité du bâti" affectées de leur coefficient de pondération $(0,19$ et 0,73$)$. Celle-ci montre que dans la ville d'Anyama, en termes de surface occupée, il y a un déséquilibre dans la répartition des enjeux matériels (31\% de faibles, près de $29 \%$ de moyens et $40 \%$ d'importants). Les zones où les enjeux sont importants sont plus étendues et correspondent entièrement ou en partie aux quartiers anciens que sont Gare, Schneider, Zossonkoi, Palmeraie et Christiankoi (Figure 5). En effet, c'est dans ces quartiers où l'on trouve encore les veilles maisons faites de banco ainsi que les constructions précaires que les lots sont densément bâtis. Tout ceci fragilise l'habitat.

Le croissement de la couche "enjeux matériels" avec celle des "enjeux humains" a permis d'élaborer la carte de tous les enjeux (figure 6). Celle-ci modifie profondément les données précédentes, en ce sens qu'en couvrant $58 \%$ de l'espace urbanisé, ce sont les surfaces de niveau d'enjeux moyen qui sont les plus étendues dans la ville d'Anyama. Les seules zones où les enjeux restent importants correspondent sur la carte à l'emplacement des poches d'habitat précaire au quartier Schneider. 


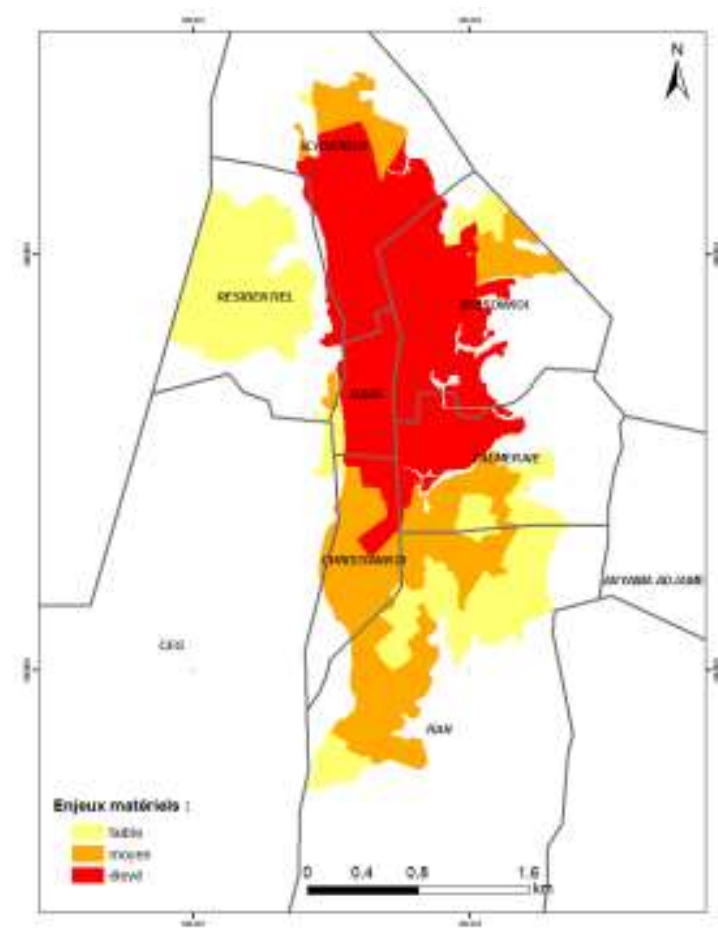

Figure 5:- Répartition des enjeux matériels dans la ville d'Anyama

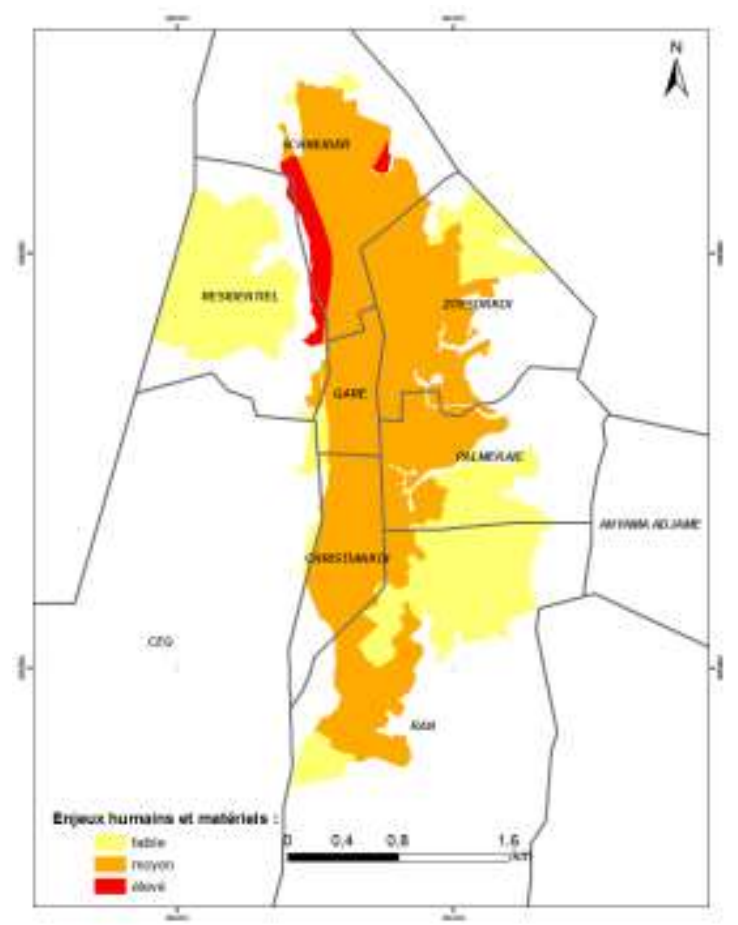

Figure 6:- Répartition des enjeux humains et matériels dans la ville d'Anyama

\section{III.3. La carte du risque d'érosion à Anyama:-}

Elle est le produit de la combinaison des couches "aléa érosion" et "enjeux humains et matériels". Il ressort de la figure 7 qu'à Anyama, les zones à risque d'érosion élevé et moyen occupent les plus grandes surfaces. En effet, $45 \%$ du site construit est exposé à un risque d'érosion élevé et $36 \%$ à un risque d'érosion moyen. En outre, les terrains soumis à un risque d'érosion élevé correspondent aux noyaux urbains anciens installés sur un relief fortement disséqué. Quant aux terrains urbains exposés à un risque d'érosion moyen, ils couvrent les zones d'extension périphérique, notamment les quartiers Palmeraie, RAN et Zossonkoi. Résidentiel est le seul quartier d'Anyama où le risque d'érosion est faible. 


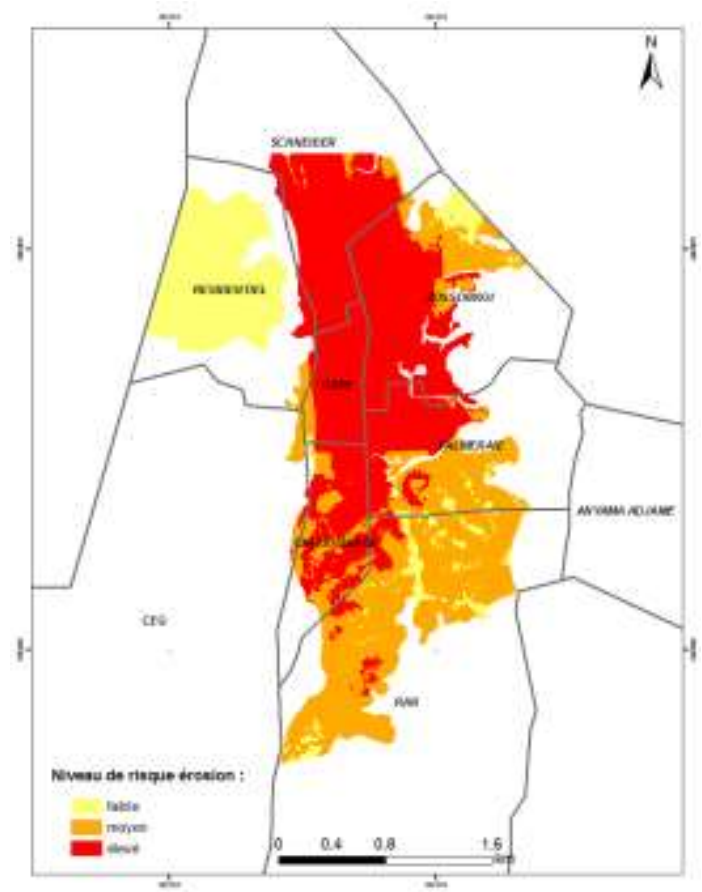

Figure 7:- Répartition du risque d'érosion dans la ville d'Anyama

\section{Discussion:-}

Les données et les techniques d'analyse utilisées dans cette étude ont permis de répondre à deux préoccupations essentielles de cet article : proposer la hiérarchisation multicritère comme une démarche fiable de cartographie du risque d'érosion et présenter cette méthode comme un outil d'aide à la décision.

Sur le premier point, les cartes établies, à partir des différents facteurs intervenant dans le processus érosif (pente, érodibilité des roches, protection du sol) ou des critères d'enjeux (densité de population, densité du bâti et qualité du bâti), ne constituent que des couches de base nécessaires à la détermination des zones à risque d'érosion. En effet, dans la nature, les trois facteurs d'aléa agissent de façon synergique, mais n'ont pas la même importance dans le développement de l'érosion. De même, les trois critères d'enjeux n'ont pas le même niveau d'exposition ou d'endommagement. Il a fallu alors définir le coefficient de pondération de chaque facteur et de chaque critère. Cette étape manque dans les quelques études menées en Côte d'Ivoire sur la répartition du risque d'érosion en milieu rural où, dès la hiérarchisation des facteurs d'érosion, les chercheurs (Kouadio, 2001 ; N'Go 2005 ; etc.) élaborent des cartes de risque d'érosion. Ces documents aussi bien élaborés soient-ils ne reflètent pas la réalité, non seulement parce qu'il s'agit là de cartes d'aléa et non de risque, mais aussi parce que ces cartes considèrent que tous les facteurs ont la même importance dans le processus érosif, ce qui n'est pas vrai.

Dans tous les cas, au cours de la pondération des facteurs ou des critères, les appréciations doivent s'appuyer sur les travaux de terrain et sur la culture scientifique du chercheur, voire une parfaite connaissance des phénomènes étudiés et des facteurs qui interviennent dans leur développement. Par exemple, pour apprécier l'aléa érosion en Côte d'Ivoire, les travaux de Roose E. $(1977,1984,1990)$ donnent des résultats incontestables. Ils montrent que dans l'aléa érosion, le facteur "couvert végétal" est de loin le plus important (Roose, 1984). Cette prééminence de la couverture végétale dans le développement de l'érosion est confirmée dans cette étude où le croisement de la carte de sensibilité (figure 3) avec la protection du sol modifie nettement la répartition des niveaux d'aléa érosion dans la ville d'Anyama (figure 4).

Concernant les enjeux, après discussions avec d'autres collègues, nous avons convenu de privilégier dans cette étude la sécurité de l'homme qu'il faut assurer, en nous inspirant des remarques faites par Dauphiné (2001). Ainsi, pour que l'homme soit affecté dans le cas de l'érosion, il faudrait que la maison qui le protège soit détruite par l'aléa. D'où l'importance accordée à la qualité du bâti à l'intérieur de laquelle l'habitat précaire est le plus vulnérable. Cela voudrait dire qu'au départ, il faut savoir ce que l'on veut mettre en évidence. 
En outre, la comparaison par paire des facteurs ou des critères relève d'un exercice intellectuel qui fait intervenir si nécessaire plusieurs chercheurs. Dans la mesure où le jugement que l'on porte sur l'importance d'un facteur ou d'un critère par rapport à un autre est subjectif, les valeurs attribuées aux jugements de deux chercheurs ne sont pas forcément les mêmes, mais elles doivent être soutenues par un raisonnement cohérent. A ce niveau, l'indice de cohérence permet de corriger les erreurs d'appréciation (lorsqu'il est supérieur à $10 \%$ ) et de rester dans une fourchette acceptable.

Le second point sur lequel nous insistons est tout aussi important que le précédent. Effet, si la superposition des différentes couches peut permettre dans tous les cas de réaliser des cartes de répartition du risque d'érosion, le message communiqué n'est pas toujours le même. Dans le cas de simples superpositions de couches, la carte résultante permet de connaitre uniquement la répartition de l'érosion en fonction du niveau de risque encouru. Avec l'analyse multicritère, la carte devient un moyen de prise de décision. En effet, à l'étape de la pondération, il est déterminé la priorité de chaque facteur d'aléa ou de chaque critère d'enjeu. L'intérêt de cette priorité est de permettre aux décideurs, dans leurs tentatives de réduction du risque d'érosion, de savoir sur quel facteur ou critère agir. La présente étude indique que, dans le cas de l'érosion à Anyama, il faut agir soit sur la protection du sol dont la priorité, c'est-à-dire la contribution dans le processus érosif, est de $71 \%$, soit sur la qualité du bâti ayant une priorité de $73 \%$, en améliorant les matériaux de construction.

Plusieurs chercheurs qui s'intéressent à la cartographie de l'érosion vont dans ce sens, en utilisant diverses méthodes multicritères pour faire face à un problème décisionnel à référence spatiale. C'est le cas de Pouliot et $a l$. (1993) qui ont utilisé la pondération des critères pour aider à l'identification des zones à haut risque d'érosion dans le bassin-versant de Merguelli (Tunisie). Il en est de même des méthodes exposées par Amor Laaribi (2000) et de la modélisation de l'aléa érosion des sols, dans le département de l'Aisne (France) établie par Le Bissonnais Y. et al. (2004).

\section{Conclusion:-}

Le modèle présenté ici est une adaptation de la Méthode de Hiérarchie Multicritère de Saaty à l'étude de la répartition de l'érosion, en milieu urbain. Il a été appliqué à la ville d'Anyama, en intégrant dans un système d'information géographique des couches d'informations issues de l'analyse des données naturelles et humaines disponibles pour la zone d'étude.

Les résultats indiquent, à l'échelle de la ville d'Anyama, les tendances de la répartition spatiale de l'aléa érosion, des enjeux et finalement du risque d'érosion. Ils permettent également d'identifier les facteurs responsables de la distribution de l'aléa et des enjeux. Ce qui est encore plus fondamental, c'est que par la connaissance de la contribution de chaque facteur au processus érosif ou à l'exposition à l'aléa, ce modèle apparait comme un véritable outil de prise de décision, dans le cadre des actions de lutte contre l'érosion des sols.

Dans la mesure où on s'est rendu compte que la répartition du risque d'érosion à Anyama est plus accentuée dans les quartiers anciens, à cause de la dénudation des sols, et qu'elle varie aussi avec la qualité de l'habitat, une réactualisation du modèle après la mise à jour des données de terrain sera nécessaire. Cela permettra de suivre l'évolution spatiale des zones à risque d'érosion et de mesurer l'impact de l'amélioration ou non du paysage urbain. Enfin, on pourrait appliquer la démarche multicritère aux villes ivoiriennes et africaines pour lesquelles les données d'entrée seraient disponibles. Une telle perspective permettra d'aider les gestionnaires urbains à prendre des décisions justes et à agir par anticipation, dans le cadre de la gestion préventive des risques naturels auxquels les villes d'Afrique subsaharienne sont de plus en plus exposées.

\section{Références bibliographiques:-}

1. AKRÉ A. M., 2002 : Urbanisme opérationnel. Yamoussoukro, Institut National Polytechnique Félix Houphouët-Boigny, 38 pages.

2. ALLA Della A., 2012 : Anyama : une ville d'érosion, in Perspectives de la géographie en Afrique subsaharienne. Actes de colloque tome 1, Abidjan, 14-17 septembre 2009. Sarrebruck (Allemagne), Éditions Universitaires Européennes, pp. 117-128.

3. AMOR Laaribi, 2000 : SIG et analyse multicritère. Hermès Science Publications, Paris, 190 pages.

4. ATSÉ Jean-Pierre, 2001 : Géomatique et gestion des risques urbains : vers des progrès attendus, in Gestion spatiale des risques. Paris, pp 25-45. 
5. DAUPHINÉ André, 2001 : Risques et catastrophes. Observer, Spatialiser, Comprendre, Gérer. Paris, Armand Colin, Coll. U, 264 pages.

6. KOUADIO Boyossoro H., 2001 : Insécurité climatique et géorisques en Afrique de l'Ouest : apport des systèmes d'information géographique et de la télédétection à l'étude des phénomènes de risques naturels dans la région semi-montagneuse de Man (Ouest de la Côte d'Ivoire). Thèse Doctorat $3^{\text {ème }}$ cycle (Hydrogéologie- environnement), Université d'Abidjan-Cocody, Abidjan, 167 pages.

7. LE BISSONNAIS Y., DUBREUIL N., DAROUSSIN J. et M. GORCE, 2004 : Modélisation et cartographie de l'aléa d'érosion des sols à l'échelle régionale : Exemple du département de l'Aisne. Étude et Gestion des Sols, Volume 11, 3, pp 307-321

8. MANCHE Y., 2000 : Analyse spatiale et mise en place de systèmes d'information pour l'évaluation de la vulnérabilité des territoires de montagne face aux risques naturels. Thèse Doctorat (Géographie), Université Joseph Fourier, Grenoble I, 172 pages.

9. N'GOH Yao A., 2000 : Etude de l'érosion des sols de la région de Buyo : analyse des facteurs et éssai d'évaluation des risques par la télédétection et les systèmes d'information géographique. Thèse Doctorat $3^{\text {ème }}$ cycle (Hydrologie et système d'information géographique), Université d'Abobo-Adjamé, Abidjan, 155 pages.

10. PAIN M. 1984 : La ville, facteur de crise écologique, in Kinshasa, la ville et la cité. Études urbaines, Paris, ORSTOM, mémoires $n^{\circ} 5$, pp. 59-72.

11. PEYROT B., 1984 : Facteurs et processus de dégradation du site de Brazzaville. Travaux et Documents du CEGET, $\mathrm{n}^{\circ} 51$, pp 113-128.

12. POUILOT J., THOMON K.P.B., CHEVALIER J.J. et BOUSSEMA M.R. (1993) : Utilisation des données

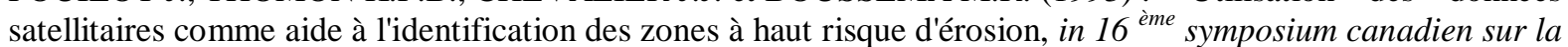
télédétection. Sherbrooke, juin, pp. 515-520.

13. ROOSE É., 1977 : Érosion et ruissellement en Afrique de l'Ouest. Vingt années de mesures en petites parcelles expérimentales. Abidjan, ORSTOM, 108 pages.

14. ROOSE É., 1984 : Impact du défrichement sur la dégradation des sols tropicaux. Machinisme Agricole Tropical, $\mathrm{n}^{\circ} 87$, pp. 24-36.

15. ROOSE É., SARRAILH J.-M., 1990 : Érodibilité de quelques sols tropicaux. Vingt années de mesure en parcelles d'érosion sous pluies naturelles. Cahiers ORSTOM, série pédologie, vol. xxv, n 1-2, pp. 7-30.

16. SAATY L. Th., 1984 : Décider face à la complexité. Une approche multicritère d'aide à la décision. Collection Université-Entreprise, Les Éditions ESF, 231 pages.

17. TCHOTSOUA M. et BONVALLOT J., 1995 : Crise socio-économique et érosion accélérée à Yaoundé : une contribution à la gestion de l'environnement urbain en milieu tropical humide, in Environnement humain de l'érosion. Actes du colloque de Paris du 20 au 23 septembre 1994, Réseau Erosion, Bull. n 15, pp. $214-231$.

18. TCHOTSOUA M. et BONVALLOT J., 2000 : L'érosion urbaine au Cameroun : processus, causes et stratégies de lutte, in influence de l'homme sur l'érosion. Actes du colloque international de Yaoundé du 09 au 19 décembre 1999, Réseau Erosion, Bull. n²0, pp. $\quad 324-331$. 